\title{
La biblioteca digital y la educa- ción a distancia como entes in- separables para incrementar la calidad de la educación
}

\author{
Brenda Cabral Vargas *
}

Artículo recibido:

24 de agosto de 2006.

Artículo aceptado:

7 de abril de 2008.

\section{RESUMEN}

El presente trabajo aborda la biblioteca digital como un elemento indispensable para la educación a distancia, y plantea el modo en que dicha biblioteca puede incrementar la calidad de esta modalidad de la educación. Se muestran además las actividades escolares que pueden ser mejoradas con el uso de la biblioteca digital. Los planteamientos construidos en este artículo se sustentan primeramente en una investigación documental sobre los distintos elementos que interactúan en la educación a distancia, pero también se apoyan en un análisis crítico de varias modalidades a distancia analizadas. El objetivo de este trabajo tiene tres vertientes: la primera es mostrar a la biblioteca digital como un espacio

* Centro Universitario de Investigaciones Bibliotecológicas de la UNAM, México. brenda@cuib.unam.mx

INVESTIGACIÓN BIBLIOTECOLÓGICA, Vol. 22, Núm. 45, mayo/agosto, 2008, México, ISSN: 0187-358X. pp. 63-78 
educativo; la segunda se refiere a los diferentes aspectos que deben considerarse para lograr que la biblioteca sea la columna medular de este tipo de modalidad educativa, y la tercera es proponer elementos que permitan mejorar la calidad de la educación a distancia a través de su interacción con la biblioteca digital.

Palabras clave: Biblioteca digital; Educación a distancia; Calidad de la educación.

\begin{abstract}
Digital Library and Distant Education as inseparable entities to better quality of education

Brenda Cabral Vargas

Digital library is shown as an indispensable element in distant education, and as a way to improve the quality of this type of education. This paper is based in documentary research but also supported in some benchmarking. The objective is three folded: a) to show digital library as an educational space; b) to refer to the different aspects which may be taken into account in order to make this type of library the central column in this educational modality, and c) to propose some scholar activities that could be improved with the use of a digital library.
\end{abstract}

Keywords: Digital Library; Distant Education; Quality of Education.

\title{
INTRODUCCIÓN
}

T a educación a distancia, comparada con la tradicional, presenta una serie de ventajas tanto para los educandos como para las instituciones educativas: la flexibilidad de horarios, en el primer caso, y la optimización de recursos, en el segundo. En el ámbito nacional, lo mismo que en el internacional, la tendencia a prescindir del contacto presencial de educandos y educadores sigue en aumento. La tecnología avanza a pasos agigantados y con ello se hace patente la necesidad de contar con diferentes formas de educación que aprovechen sus bondades. En este contexto, el buen uso de la 
tecnología ofrece la posibilidad no sólo de impartir cursos a distancia, sino de encontrar en un solo lugar la información y recursos didácticos que apoyen la instrucción, a lo cual puede accederse de una manera rápida, lógica y didáctica, que transforme dicha información en aprendizaje significativo para los usuarios de dicho espacio educativo.

El interés por conocer cuál es la trascendencia de la participación de las Bibliotecas Digitales (BD), que apoyan la formación intelectual de los alumnos de las modalidades a distancia y las relaciones que las unidades documentales tienen con los docentes, investigadores y alumnos, motivan este trabajo de investigación.

\section{La biblioteca digital como espacio educativo}

La BD es un espacio educativo por excelencia, un lugar donde se entrelazan la información y los conocimientos; un medio para que el trabajo colaborativo fructifique; un sitio en el que se encuentran recursos informativos, contenidos didácticos y los usuarios, además de los "bibliotecarios digitales"; es decir, el personal profesional que en un ámbito virtual cumple las funciones de un bibliotecario tradicional en la institución análoga (Biblioteca Presencial o BP), conjunto que genera nuevos conocimientos en la interacción entre los medios y la información.

\section{Cómo lograr este escenario}

Para que este escenario pueda tornarse en una realidad, una BD debe tener, además de colecciones digitales desarrolladas expresamente para satisfacer las demandas de información de la comunidad a la que sirve, servicios y personal especializado en sus ámbitos de competencia. En otras palabras, una $\mathrm{BD}$ debe ofrecer los mismos servicios que una BP, pero además incluir otros servicios que sólo se obtienen a través del buen uso de la tecnología, y entre los cuales se encuentran los siguientes:

- La localización y obtención de documentos.

- Boletines bibliográficos.

- La sección de venta de libros de la propia institución a la que pertenece la biblioteca digital.

- Ayuda a los usuarios cuando éstos no localicen la información o no puedan acceder a algún recurso de la BD.

- Herramientas de instrucción a usuarios, los cuales incluirían tutoriales interactivos. 
A simple vista, los servicios propuestos no parecen diferentes a los de las $\mathrm{BP}$, sólo que en este caso deben ser más personalizados; es decir, desarrollarse expresamente de acuerdo con las necesidades de sus usuarios, por lo cual deben responder a intereses particulares o perfiles predeterminados, conocidos de antemano por el bibliotecario.

Además de lo anterior, algunas actividades técnicas y administrativas de las bibliotecas se pueden mejorar al incluir espacios como:

- Apartados en los que se indiquen cuáles son las políticas de selección y adquisición de la BD.

- El fomento de la donación de recursos electrónicos como tesis, documentos internos de trabajo o investigación que puedan generar un apartado como documentos no publicados en algún otro medio. Esta sección hará las veces de un archivo vertical, sólo que en este caso será digital. Para ello, el donador y autor del trabajo deberán firmar un escrito en el que cedan sus derechos de propiedad a la BD para que ella pueda organizar, analizar, difundir y procesar dicha información.

\section{Colecciones}

Hablábamos antes de las bondades de la tecnología y cómo ésta hace posible, de manera cada vez más sencilla, la oferta de contenidos digitales diferentes a los libros y revistas electrónicas, como son obras de consulta, publicaciones oficiales y otro tipo de materiales que pueden constituir una mediateca (en la que pueden incluirse videos, gráficas, mapas, archivos de sonido, programas interactivos y todo tipo de archivos multimedia).

Para poder incrementar la calidad en la educación a distancia, la BD debe mostrar todos los servicios que ofrece la institución educativa a la que corresponde para apoyar a la plantilla académica (maestros, alumnos, investigadores, etcétera) que pertenece a una modalidad a distancia. Esto entre otras cosas, convertirá a la BD en la puerta de acceso para las asignaturas y módulos de la educación a distancia, a través de sus recursos de información. De esta manera, la BD se erigirá y no sólo en una herramienta de aprendizaje, sino en un espacio de aprendizaje en el que la educación a distancia no podrá ser concebida sin ella y prometerá "[...] dar nuevas vías a la educación para permitir su avance en relevancia, pertinencia, calidad y cobertura".

1 Alejandro Pisanty Baruch, "Distancia y presencia : nuevos paradigmas en educación a distancia", en El medio digital en el siglo XXI : retos y perspectivas para los bibliotecólogos, investigadores, educadores, y editores [Disco Compacto] / Angélica Rosas Gutiérrez, comps. Georgina Araceli Torres Vargas, México, CUIB, 2001, p. 121. 
Veamos el siguiente cuadro, en el que se muestran las relaciones existentes entre dichos elementos:

Biblioteca digital $\longrightarrow$ Contenidos $\longrightarrow$ Educación a distancia

Biblioteca digital $\longrightarrow$ Servicios $\longrightarrow$ Educación a distancia

Biblioteca digital $\longrightarrow$ Personal $\longrightarrow$ Alumnos

La BD proporciona los insumos necesarios para que una modalidad a distancia funcione de manera óptima. El quehacer del bibliotecario se ha sofisticado: se ha ampliado su ámbito laboral y la temática de sus investigaciones, a lo cual tiene que actualizarse el profesional de la información para superar su imagen tradicional, así como para desarrollar aptitudes relacionadas con los métodos, procedimientos y estrategias de investigación que lo lleven al aprovechamiento de las tecnologías de la información y a proporcionar no sólo información sino también conocimiento a los usuarios.

Como veremos en el siguiente dibujo, la BD quedaría en el centro de los elementos que contribuyen al proceso enseñanza/aprendizaje en una modalidad a distancia.

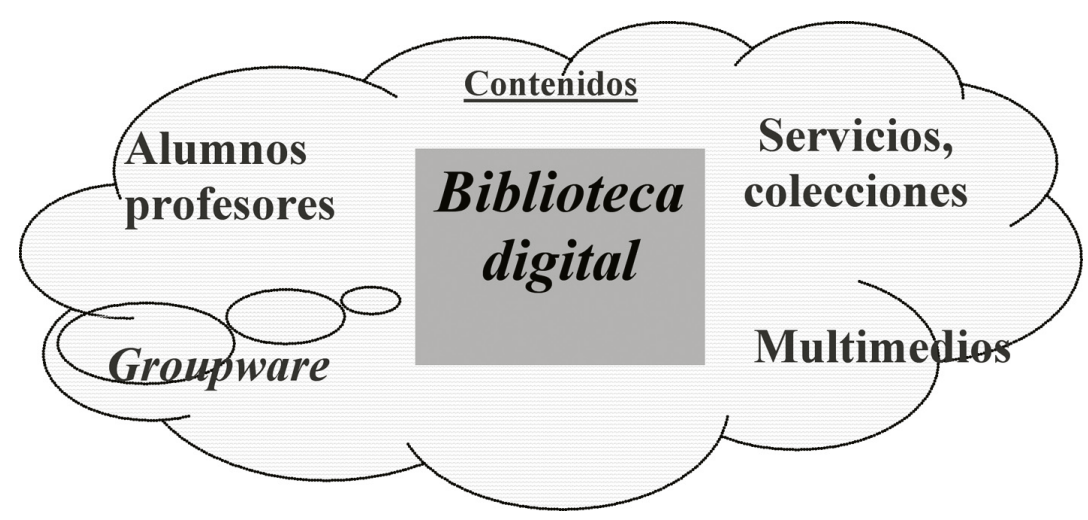

Para desarrollar el aprendizaje significativo, los diferentes elementos deberían interactuar en diferentes momentos y en diversas situaciones, como las siguientes:

- Cuando un alumno acude a consultar material como apoyo a sus clases.

- Cuando el profesor interactúa con sus alumnos a partir de la discusión de algunas fuentes bibliográficas. 
- Cuando el profesor deja material interactivo que el alumno debe revisar y, posteriormente, lleva a cabo algunas actividades de evaluación para ver si se logró el aprendizaje a través de los multimedios.

Una adecuada noción sobre el ambiente de aprendizaje supone pensar en una serie de espacios en los que la comunicación toma un lugar superior y ayuda a asimilar cómo debe usarse la tecnología de manera adecuada; así como la forma en que deben ser tratados los materiales antes de ser puestos como material de apoyo o didáctico en las modalidades a distancia; y por otro lado impulsa la integración de equipos responsables de estas tareas en las instituciones educativas.

\section{LA BIBLIOTECA DIGITAL Y EL DESARROLLO DE LOS CONTENIDOS}

La biblioteca digital es el espacio ideal que puede brindar materiales didácticos de una manera más cercana y conveniente a las necesidades de los usuarios, y es por ello que se establece un vínculo inseparable entre ésta y los contenidos didácticos, los medios y los materiales de aprendizaje; que son algunos de los elementos indispensables de la educación a distancia. Se habla de un espacio ideal porque la BD permite la interacción entre los usuarios, los docentes y los contenidos, facilitando la colaboración, además de fomentar la investigación y la generación de nuevos conocimientos, por mencionar sólo algunas de sus aportaciones a la educación a distancia.

Los medios y materiales de los que antes hablábamos son definidos por Chan como el

[...] resultado de un proceso de diseño y producción, en el que intervienen profesionales de diversas áreas para la presentación de un curso o unidad de aprendizaje. Son por lo tanto productos comunicativos abiertos. ${ }^{2}$

Es decir, se espera que los estudiantes trabajen con la información no como si ésta fuera un discurso acabado, sino como un insumo para su propia producción discursiva. También se menciona que "[...]los medios se gestionan y operan como sus canales de distribución bidireccional", lo cual quiere decir que si los textos son abiertos e inacabados, más que la consideración de

2 María Elena Chan Núñez, "Los ambientes y materiales en el diseño de ambientes de aprendizaje en la educación a distancia”, en El medio digital en el siglo XXI : retos y perspectivas para los bibliotecólogos, investigadores, educadores, y editores [Disco Compacto] / Angélica Rosas Gutiérrez, comps. Georgina Araceli Torres Vargas, México, CUIB, 2001, p.100. 
cómo se hace llegar el mensaje primario que emite el educador, preocupa el canal a través del cual el educando emitirá su propio mensaje. El desarrollo de medios supone la consideración de ambas vías.

Con esta premisa podemos asegurar que la BD en este proceso es, al mismo tiempo, el objeto y el espacio de aprendizaje ideal para fomentar la colaboración, el intercambio y la generación de nuevos conocimientos, que apoyen a su vez a la educación a distancia.

La BD facilita el desarrollo de contenidos para ser expuestos en la educación a distancia en tanto que permite que la exposición de los temas de las diferentes asignaturas pueda ser arreglado de una manera lógica, disciplinar y pedagógica; es decir, adecuada al nivel y al tipo de estudios, pero siempre, como ya se mencionó, sin que estos documentos estén fijos o acabados. Esto les permitirá a los estudiantes recuperar, entre otras cosas, las relaciones de pertinencia, jerarquía y secuencia entre los conceptos de una disciplina; comprender las implicaciones operativas de un enfoque teórico y apreciar la eficacia de algunos procedimientos metodológicos; así como participar consciente y activamente en la construcción de su conocimiento.

La concepción de acceso es una cuestión interesante, ya que cuando se analiza cómo los individuos pueden acceder a las bibliotecas digitales, lo que generalmente entra en debate es el problema de la omisión, porque las personas que no cuentan con tecnología quedan alejados del privilegio de pertenecer a una comunidad educativa, de conocer más sobre aspectos sociales, culturales, laborales, etcétera.

En muchos casos los alumnos de educación a distancia no tienen acceso a una BD por causas que están más allá de la carencia del equipo, puesto que cuando es necesario puede acudir a un servicio público y rentarlo; de hecho, la modalidad a distancia no sería posible para alguien que no pueda subsanar esta carencia. Uno de los principales obstáculos es un "[...]complejo entramado de conocimientos necesarios para considerar que se tiene acceso a la tecnología".

Para Callister y Burbules este debate no debería quedarse en el aspecto técnico, sino extenderse a la oportunidad de desarrollar las aptitudes y las actitudes que son necesarias para aprovechar el recurso. Lo anterior supone la necesidad de que los estudiantes desarrollen una serie de habilidades que les permitan explotar los recursos que les ofrece tanto la modalidad a distancia como la BD, y dichas habilidades no tienen que ver sólo con las cuestiones

3 N. L. Dari, "Entre riesgos y promesas: Educación digital", [reseña de libro: educación: Riesgos y promesas de la nueva tecnologías de la información], en Revista Electrónica de Investigación Educativa, 6(2), (2004), http://redie.uabc.mx/vol6no2/contenido-dari.html [consulta: el 26 de mayo del 2006]. 
técnicas del funcionamiento del sistema de educación, sino con el raciocinio necesario para que el estudiante pueda hallar por sí mismo, con la mínima ayuda, la información que necesita y la cual ya se encuentra dentro de este espacio educativo.

La otra cara de la moneda corresponde a las habilidades que debe poner el bibliotecario digital. Para que los usuarios que se encuentren en una modalidad a distancia puedan hacer uso de los documentos, los recursos y la información generalmente localizada en la BD, es necesario que el bibliotecario posea la capacidad de elegir información que realmente pueda resultar útil y descartar la información que no lo es, por innecesaria, trivial y, a veces, hasta irrelevante para nuestros usuarios. Como una solución a lo anteriormente mencionado, Burbulles y Callister ${ }^{4}$ proponen que el lector se convierta en un hiperlector; es decir, aquella persona capaz de realizar lecturas de los documentos y discernir entre un documento y otro, a través del análisis del mismo. Por mi parte yo sostengo que el bibliotecario debe ser el encargado de discriminar la información poco útil pero para estar en condiciones de realizar esto tiene que encargarse de realizar una lectura activa y crítica; esto es, convertirse en un hiperlector, y hacerse poseedor de una formación enciclopédica.

Además, al ofrecerles a sus usuarios una serie de recursos informativos, la BD permite acudir a fuentes de información que complementan lo aprendido con las actividades de aprendizaje, y al mismo tiempo brinda espacios en los que se puede comentar, corregir, ampliar y complementar los recursos didácticos ya existentes.

Actualmente se realizan estudios relacionados con los documentos multimediales, que consisten en una propuesta de lectura no lineal que modifica la relación texto-imagen, texto-texto y texto-lector. En esta propuesta, el texto en pantalla ya no es tan abundante pero, no por ello es menos significativo. Las imágenes fijas y/o en movimiento y el sonido (como la voz del profesor) adquieren gran importancia y fungen como guías e ilustran el contenido, creando la sensación de tener al profesor en casa. Además, se debe de evitar la creencia de que un documento debe "[...] ser lineal y sujetar al lector a una forma de lectura y revisión predeterminada por autores y editores". ${ }^{5}$ Por el contrario, los documentos digitales permiten diversas formas de navegación en la información contenida y una amplia gama de opciones para abordar

4 Nicholas Burbules, y Thomas Callister, Educación: Riesgos y promesas de las Nuevas Tecnologías de la Información, Argentina, Editorial Gránica, 2000, p.3.

5 Ramiro Lafuente López, [et.al], "La enseñanza de la bibliotecología digital en la modalidad de educación a distancia”, en XXXI Jornadas Mexicanas de Biblioteconomía, 31 de mayo y 1 y 2 de junio del 2000, p.55. 
la lectura por parte de los usuarios, en cuanto a que no lo circunscriben a un solo documento sino a variadas formas de relación entre los contenidos de distintos documentos, aspectos que deben ser considerados al desarrollar contenidos para programas de esta naturaleza.

Lo que rige, entonces, es el texto que llama a otros textos (hipertextos) cuando sea conveniente o necesario ampliar significados. En este sentido, el papel de la BD cambiará dejando de ser una contenedora de información y se convertirá en un espacio virtual de aprendizaje y generación de nuevos conocimientos.

Un aspecto al parecer olvidado de la BD es la investigación. En un estudio llevado a cabo por Marisol Ramírez, del Instituto Tecnológico y de Estudios Superiores de Monterrey (ITESM), campus Juárez, sobre la forma en que se usa la tecnología en la educación, observa la autora que la mayoría de los programas de educación a distancia no fomentan esta actividad tan importante dentro de la educación superior. Una cifra alarmante es que el $83 \%$ de esos programas no efectúa investigación, en tanto que sólo el 17\% sí la considera como actividad de aprendizaje o como parte de la formación de los estudiantes a distancia. ${ }^{6}$

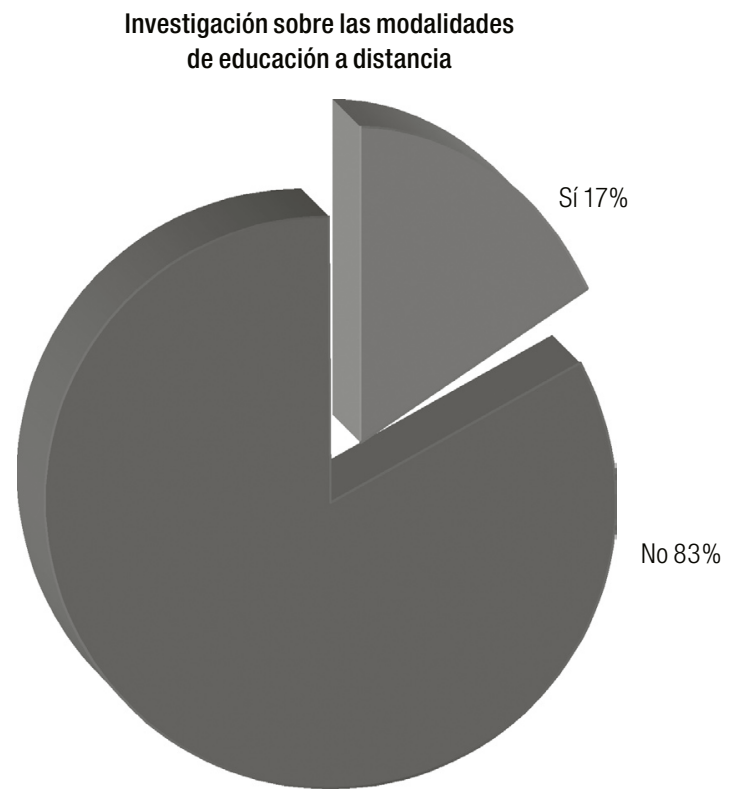

6 Marisol Ramírez, Acercamientos a los ambientes virtuales en México : Investigación sobre el uso de las tecnologías en la educación a distancia, México, ITESM, 2002. 
La BD puede fomentar la investigación en las instituciones educativas que tienen la modalidad de educación a distancia si toma en cuenta lo siguiente:

- Difundir artículos o textos de interés para sus usuarios.

- Establecer foros de discusión sobre temas de interés relacionados con el área.

- Fomentar la colaboración e integración en equipos de trabajo entre sus usuarios con algunos expertos o centros que manejan temas afines.

- Permitir que algunos servicios de información envíen sus servicios de alerta o que puedan difundir sus investigaciones a través de los títulos de revistas generados por las empresas dedicadas a la venta de servicios de información.

La relación existente entre la BD y la educación a distancia es múltiple y, según Burbules y Callister existen tres planteamientos entre la tecnología de la información y la educación:

[...]el primero objeta la manera de caracterizar como tecnologías de información a algunas de ellas. El segundo propone una concepción relacional de la tecnología y el último sostiene una postura postecnocrática en materia de políticas públicas, todo esto como un punto de partida para reflexionar sobre las condiciones y motivaciones de la nueva tecnología para la enseñanza y el aprendizaje. ${ }^{7}$

En relación con el primer planteamiento, si bien la BD está inmersa en las tecnologías de información (transmisora de hechos, datos, información, etcétera), dicha información no está libre de riesgos o de ideología, sino que, por el contrario, ya ha sido seleccionada, filtrada e interpretada por otros. Pero también la $\mathrm{BD}$ es un espacio de comunicación, ya que en este ir y venir de la información hay así un movimiento de lenguaje que define sus propias finalidades y normas. Por último, el tercer cuestionamiento tiene el propósito de mostrar que la BD se encuentra en un entorno, es decir, un ciberespacio donde se producen interacciones humanas peculiares y diferentes del entorno cotidiano presencial. Reconocer estas características sirve para darnos cuenta que la BD, en particular la académica, se configura en un territorio potencial de colaboración que puede impulsar otras variantes de la actividad de enseñanza/aprendizaje, no

7 N. L. Dari, “Entre riesgos y promesas: Educación digital”, [reseña de libro: educación: riesgos y promesas de la nueva tecnologías de la información], en Revista Electrónica de Investigación Educativa, 6(2), 2004, p.2 http://redie.uabc.mx/vol6no2/contenido-dari.html [consulta: el 26 
mejores o peores, sino lógicamente diferentes y que pueden apoyar la educación a distancia en toda la extensión de la palabra.

De lo anterior se desprende que

[...]la creación de nuevos ambientes de aprendizaje exige situaciones educativas en que se propicie el contacto, el intercambio y la participación de cada miembro de un grupo independientemente del espacio y tiempo, por lo que pueden ser presenciales o a distancia, sincrónica o asincrónica. ${ }^{8}$

Ahora bien, si hablamos de la colaboración para lograr aprendizaje, varios autores, como Felder, ${ }^{9}$ Timothy Butler and David Coleman, ${ }^{10}$ Landsberger, ${ }^{11}$ Roberts $\operatorname{Tim}^{12}$ (2004), han estudiado la manera como con la mencionada dicha actividad de aprendizaje se logra una mayor fijación de lo aprendido. Pero para que esto se dé la BD deberá ser consciente de ello, con lo cual podrá participar activamente apoyando con dichas actividades y, por ende, alcanzar una mayor calidad en la educación a distancia. La pirámide que se muestra en la siguiente página señala de manera gráfica las actividades de aprendizaje que permiten una mayor fijación.

8 Sistemas Telemáticos para la educación continua, México,: IPN, 1999, p.10.

9 Richard M. Felder, Aprendizaje cooperativo en cursos técnicos: procedimientos, dificultades y recompensas [en línea], North Carolina State University \& Rebecca Brent, East Carolina University. http://www.ncsu.edu/felder-public/Cooperative_Learning.html [consulta: 28 de mayo del 2006].

10 Timothy Butler and David Coleman. Models of Collaboration, 2003, [en línea] http://www. collaborate.com/publication/newsletter/publications_newsletter_september03.html [consulta: 23 de junio del 2006].

11 Joe Landsberger, El sitio Estudio: Guías y Estrategias (Study Guides and Strategies, 2005, [en línea] http://www.studygs.net/espanol/cooplearn.htm [consulta: 21 de junio del 2006].

12 Tim Roberts, Online collaborative learning in higher education, [en línea]. http://clp.cqu. edu.au/index.htm [Consulta: 26de julio del 2006]. 


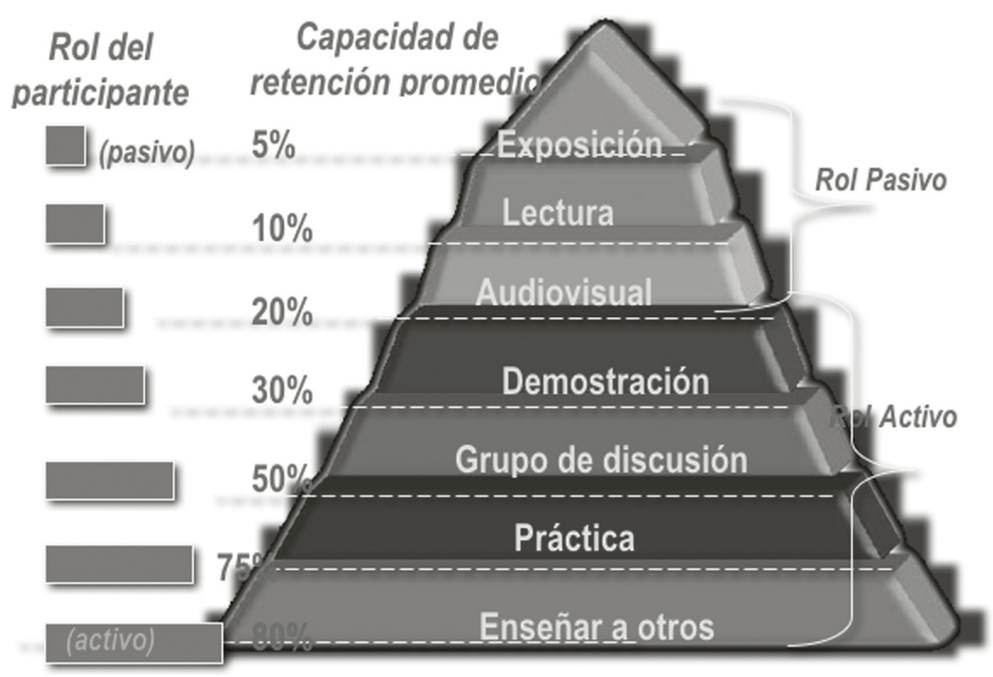

Fuente: Los porcentajes y datos fueron obtenidos de: Bethel, Maine, Pirámide del aprendizaje. U.S.A.: National Training Laboratories, [en línea], http://www.archivos.alergia. org.ar/12002/aplicacion.pdf

La pirámide nos evidencia cómo la actividad de enseñar a otros, junto con la práctica, permite que la retención de los conocimientos se lleve a cabo de una manera más adecuada y, por ende, que la calidad del aprendizaje sea mejor, pues no basta con aprender a corto plazo; es preferible lograr conservar en la memoria información que va a servir más adelante para tomar decisiones y resolver problemas de diversa índole, tanto profesionales como personales.

La calidad educativa se instala en los subprocesos que incorporan y articulan recursos para un uso inteligente,

[...]lo que significa que demuestran ser satisfactorios por que son útiles, valiosos, viables, precisos, realistas, lúcidos, prudentes y éticos, y, por ello, se han incorporado al hardware, software y al mindware (o tecnologías invisibles) ${ }^{13}$

de la práctica social y educativa.

El bibliotecario digital, además de brindar la información requerida por los usuarios, debe ser un facilitador entre los recursos informativos y el

13 B. Fainholc, "El conocimiento tecnológico necesario en este fin de siglo: acerca de la necesidad de las tecnologías invisibles", Ponencia presentada en SOMECE 97, en XIII Simposio Internacional de Computación en la educación, del 20 al 24 de set/1997, Toluca, México, 1997. 
aprendizaje, pero debe convertirse también en un guía para que el alumno de este tipo de modalidad pueda aprender de manera autodidacta, y para fomentar el autoaprendizaje. Es importante también concienciar a los usuarios sobre la necesidad de entrar a la "[...] nueva vertiente de la educación, o nueva conciencia de vertiente de la educación, que tiene que ver con el aprendizaje a lo largo de la vida". ${ }^{14}$

La globalización es otro aspecto que debe considerar la BD para fomentar la calidad en la educación, ya que este fenómeno está acarreando cambios de entorno a los cuales hay que irse incorporando de una manera creativa, y no sólo acatar lo que viene de afuera, pues mediante la educación se debe adquirir el poder de introducir modificaciones y tomar lo bueno de la globalización, que puede ser la multiplicidad cultural y educativa.

La BD debe ser un elemento de apoyo inseparable e insustituible de las modalidades a distancia para que los usuarios aprendan todo lo que necesitan y que lo hagan por el camino que más les llame la atención y mediante las estrategias que les sean más pertinentes; puesto que los adultos por lo regular ya tienen generado un estilo de aprendizaje, es decir una forma de asimilar los conocimientos que hay que aprovechar. Con esto estaríamos propiciando la flexibilidad, que es un aspecto que se maneja en la política educativa nacional y que debe ser considerado por todas las instituciones académicas de nuestro país. ${ }^{15}$

Finalmente es evidente que el papel de la BD es muy complejo y que no se concreta a ser una herramienta de trabajo de las modalidades a distancia sino que es un elemento primordial e integral para aprender. Con esto podemos decir que la BD es el espacio, el objeto, los recursos y los contenidos, necesarios todos ellos para que se realice el proceso de aprendizaje de manera adecuada. No se debe concebir el desarrollo de estos dos entes de manera separada sino en un contexto integrado, con la finalidad de elevar la calidad de los programas educativos de las modalidades a distancia.

\section{CONCLUSIONES}

El papel de la BD en la educación a distancia es cada día mayor en varios aspectos del proceso de enseñanza/aprendizaje, ya que a partir de este tipo de biblioteca se mejorará y ampliará la formación de los estudiantes puesto que:

14 Centro Interactivo Multitecnológico de Educación a Distancia, "El Poder Transformador de la educación”, en Innovación Educativa, 5 (no.27) jul-agost, 2005, p.56.

15 Jorge Petrosino, ¿Cuánto duran los aprendizajes adquiridos?: el dudoso ideal del conocimiento impecable, Jorge Petrosino, Buenos Aires, Argentina; México, Novedades educativas, 2000, p.84. 
- Serán mejores profesionales capaces de enfrentarse a los nuevos retos de búsqueda, retroalimentación y generación de nuevos conocimientos.

- Se propiciará una mayor interacción entre alumnos-docentes-contenidos.

- Habrá un mayor aprovechamiento de los contenidos por parte de los estudiantes.

- Alumnos y usuarios serán capaces de llevar a cabo diferentes investigaciones en sus áreas de interés, con lo cual desarrollarán contenidos que apoyen tanto a ellos mismos como a sus compañeros y maestros.

- Contarán con cuerpos académicos que se responsabilicen de solucionar las necesidades profesionales y sociales de sus áreas de estudio.

No se debe dejar de lado que el impacto que ejerzan las BD sobre la formación de los alumnos que estudien en las modalidades a distancia dependerá en gran medida de los contenidos, servicios y espacios que contenga dicha biblioteca, por lo que es importante tomar en cuenta la planeación de todos los elementos mencionados en este artículo.

En general, la BD será un ente sin el cual la educación a distancia no podrá ser concebida, y cuya utilización será una parte indispensable de la funcionalidad de cualquier modalidad a distancia, lo cual incrementará la calidad de dicha modalidad elevando el nivel de aprendizaje hasta llevarlo a la altura de la máxima competencia; es decir, el uso del conocimiento que transforma la realidad; la propia, la de la sociedad, la de un país y en general la de la humanidad.

\section{Obras Consultadas}

Bethel, Maine. Pirámide del aprendizaje, U.S.A., National Training Laboratories, [en línea] http://www.archivos.alergia.org.ar/12002/ aplicacion.pdf [consulta: 24 de marzo del 2006]

Burbules, Nicholas y Thomas Callister, Educación: Riesgos y promesas de las Nuevas Tecnologías de la Información, Argentina, Editorial Gránica, 2000.

Butler, Timothy y David Coleman, Models of Collaboration, 2003, [en línea] http://www.collaborate.com/publication/newsletter/publications_newsletter_september03.html [consulta: 23 de junio del 2006].

Centro Interactivo Multitecnológico de Educación a Distancia, "El Poder Transformador de la educación”, en Innovación Educativa, 5(no.27) jul-agost, 2005, pp. 55-8. 
Chan Núñez, María Elena, "Los ambientes y materiales en el diseño de ambientes de aprendizaje en la educación a distancia”, en El medio digital en el siglo XXI : retos y perspectivas para los bibliotecólogos, investigadores, educadores, y editores [Disco Compacto], Angélica Rosas Gutiérrez, comps. Georgina Araceli Torres Vargas, México, CUIB, 2001.

Dari. N. L., "Entre riesgos y promesas: Educación digital”. [reseña de libro] Educación: Riesgos y promesas de la nueva tecnologías de la información, en Revista Electrónica de Investigación Educativa, 6(2), (2004), http://redie.uabc.mx/vol6no2/contenido-dari.html [Consulta: el 26 de mayo del 2006].

Fainholc, B., "El conocimiento tecnológico necesario en este fin de siglo: acerca de la necesidad de las tecnologías invisibles", ponencia presentada en SOMECE 97, en XIII Simposio Internacional de Computación en la educación, del 20 al 24 de set/1997, Toluca, México, 1997.

Felder, Richard M., Aprendizaje cooperativo en cursos técnicos: procedimientos, dificultades y recompensas [en línea], North Carolina State University \& Rebecca Brent, East Carolina University. http://www.ncsu.edu/felder-public/Cooperative_Learning.html [consulta: 28 de mayo del 2006].

Lafuente López, Ramiro [et.al] "La enseñanza de la bibliotecología digital en la modalidad de educación a distancia", en XXXI Jornadas Mexicanas de Biblioteconomía, 31 de mayo y 1 y 2 de junio del 2000 .

Landsberger, Joe. El sitio Estudio: Guias y Estrategias (Study Guides and Strategies, 2005, [en línea] http://www.studygs.net/espanol/ cooplearn.htm [consulta: 21 de junio del 2006].

Morán Zea, Alejandro, Miguel Ángel Sánchez Villegas y Sergio Sepúlveda Horta, Administración y edición de libros en XML en la BD del ILCE, [en línea] México, Instituto Latinoamericano de la Comunicación Educativa http://scholar.google.com/scholar?hl=en $\& \mathrm{l} r=\& \mathrm{q}=$ cache: $x s m s R d G g-l w J$ :eprints.rclis.org/archive/00002961/01/ilce.pdf+aplicaciones+and+biblioteca+and+di gital [consulta. 25 de enero del 2006].

Petrosino, Jorge, ¿Cuánto duran los aprendizajes adquiridos?: el dudoso ideal del conocimiento impecable, Jorge Petrosino. Buenos Aires, Argentina ; México, Novedades educativas, 2000, 122 p.

Pisanty Baruch, Alejandro, "Distancia y presencia : nuevos paradigmas en educación a distancia”, en El medio digital en el siglo XXI : retos y perspectivas para los bibliotecólogos, investigadores, educadores, y editores [Disco Compacto] / Angélica Rosas Gutiérrez, comps. Georgina Araceli Torres Vargas. México, CUIB, 2001.

Ramírez, Marisol, Acercamientos a los ambientes virtuales en México : Investigación sobre el uso de las tecnologías en la educación a distancia, México, ITESM, 2002. 
Sistemas Telemáticos para la educación continua, México, IPN, 1999. $110 \mathrm{p}$.

Tim, Roberts, Online collaborative learning in higher education, [en línea] http://clp.cqu.edu.au/index.htm [Consulta: el 26 de julio del 2006] . 